\title{
Study on A Chinese Medicine Chewable Tablet for Anti-Alcoholism and Liver Protection
}

\author{
Shu-Yi Dong ${ }^{1}$, Jia-Yi Lin ${ }^{1}$, Ni Yan ${ }^{1 *}$ \\ ${ }^{1}$ School of Management, Hubei University of Chinese Medicine; Wuhan, China
}

\begin{abstract}
In this study, the resources of traditional Chinese medicine were exploited reasonably, which was mainly composed of the active components of malus hupehensis and four kinds of plant components, such as Pueraria lobata, tangerine peel, jujube and corn sweet core. The preparation process of the product has been solved, and preliminary product efficacy experiments have been carried out, which has been proved to be able to relieve the symptoms of alcohol discomfort.
\end{abstract}

\section{Research background}

Chewable tablet is a practical, convenient and effective form of tablet. After chewing, the surface area of the tablet increases, which can promote the metabolism and absorption of the drug in the human body. The chewable tablets can accelerate the disintegration of some hard-to-disintegrate drugs and improve the efficacy of the drugs. The use of chewable tablets is getting more and more attention because of its special advantages. At present, chewable tablets are mainly divided into calcium tablets, vitamins, acid medicine, food and health products.

At present, the research on chewable tablet at home and abroad is mainly focused on the following areas: first, the research on excipients in chewable tablet. For example, sucrose is used as a flavor corrector to prepare saturated chewable tablets; $95 \%$ ethanol is used as the adhesive of Jingangteng chewable tablets [1]; xanthocollagen is added to chewable tablets to increase the taste, etc.. The second is the study on the preparation technology of chewing tablets. For example, the hardness of chewable tablets is adjusted by selecting binders; when making chewable tablets of traditional Chinese medicine such as burdock chewing tablets, the medicinal materials are generally extracted and separated to get extracts, and then excipients are added to make soft materials. aspirin chewable tablets are also made by pressing tablets directly with powder to improve drug stability [2], etc.. The third is the study on the clinical application of chewing tablets, such as penicillin $\mathrm{V}$ potassium chewable tablets for children, Shuanghuanglian chewable tablets with low cost and little adverse reactions, and geriatric antihypertensive chewable tablets developed by NikhiL. Furthermore, chewable tablets have special value in clinical use because of their own advantages.

The chewable tablet, developed in this paper takes tangerine peel soup in General Records of Holy Universal Relief as the theoretical basis, adds and subtracts on the basis of the ancient prescription, and adds Hubei characteristic medicinal material -- malus hupehensis, to protect liver and alleviate acute and chronic liver injury by using the active ingredient-phloridin in malus hupehensis. In addition, the chewable tablet is supplemented with homologous ingredients of medicine and food, such as Pueraria lobata, tangerine peel, jujube and corn sweet core, so that it can systematically solve the discomfort after drinking, while adding the taste of the product through healthier biological sugar and bring consumers a brand-new experience. In this way, it can be considered to have broad market prospects.

\subsection{Causes of discomfort after drinking}

After entering the human body, alcohol first enters the stomach and mixes with gastric acid, enters the intestinal tract through the pylorus under the action of gastric peristalsis, and is absorbed into the blood circulation in the mucosal epithelial cells of the small intestine. After entering the circulation, $10 \%$ of the alcohol is excreted out of the body in the form of sweat, breathing, etc., and the remaining $90 \%$ goes to the body's chemical plant-liver for treatment. In the process of alcohol metabolism in the liver, the lipid components on the surface of hepatocytes are over-oxidized, resulting in the destruction of hepatocyte membrane. After further deterioration, it will produce obstacles to the decomposition and metabolism of fatty acids, resulting in intrahepatic fat deposition and the formation of fatty liver. With the increase of the amount of alcohol and the extension of drinking time, the damage of alcohol to the liver gradually developed according to the trilogy of "alcoholic fatty liver, alcoholic hepatitis and alcoholic cirrhosis".

\footnotetext{
*Ni Yan: Yan219@hbtcm.edu.cn
} 


\subsection{Research progress of anti-alcoholism food}

At present, the research on anti-alcoholism food at home and abroad is mainly focused on the following areas: first, the liver-protecting and spleen-invigorating traditional Chinese medicine health care products, which add a certain ingredient with anti-alcoholism and liver protection effects to the food. For example, in China, ingredients that are frequently added include blueberries that paly an antioxidant effect and are rich in a variety of amino acid vitamins; prickly pears that increase the body's SOD, catalase activity and reduced glutathione content; and kudzu root that promotes ethanol metabolism Vegetarian, and Pueraria lobata, which also has the effect of protecting the liver. Since ancient times, there have been many prescriptions for relieving alcoholism. Sun Simiao, Sui Dynasty, recorded a compound hangover juice with Pueraria lobata juice, celery juice, and loquat leaves as the main ingredients in Thousand Gold Pieces Formulary; Li Dongyuan, a famous medical scientist in the Jin Dynasty, recorded "Gehua Jieyang Decoction" in Treatise on The Spleen And Stomach; In the Song Dynasty, there are as many as 30 kinds of drugs that clearly record the effect of relieving alcohol in the Book of Materia Medica. At present, Pueraria lobata, Pueraria lobata and Fructus Aurantii are the most common antialcoholic ingredients in Asia[3]. In Japan and South Korea, the most common ingredient is curcumin, which is almost insoluble in aqueous solution because of its lipophilicity and is not easily absorbed by oral curcumin. The absorbed curcumin is rapidly excreted from the body by metabolism, and the bioavailability in the body is very low, which affects its direct use as a medicine[4]. While in Europe, America and Oceania, the most common ingredient is milk thistle. American researchers discovered the protective effect of milk thistle on the liver, and thus applied it to anti-alcoholism products.

Anti-alcoholism foods relieve discomfort after drinking and alleviate the symptoms of alcohol injury. With the development of the times and the awakening of people's awareness of health, anti-alcoholism foods have ushered in a better development space and market blue sea, but there are also obvious problems: first, the homogenization of product formulations is serious, and the most common ingredients of anti-alcoholism foods in China are Pueraria lobata and trifoliate fruit. Second, most of the anti-alcoholism food dosage forms are oral liquid beverages, granular candies and dietary tea bags, which are mainly convenient for people to eat. However, these products have a large number of chemical sugar use, which will increase the sugar intake of eaters, which is not conducive to health.

\subsection{Mechanism of Action of Chinese Medicine}

Malus hupehensis is a traditional plant for both medicine and food. A large number of studies have found that malus hupehensis leaves contain flavonoids mainly phloridin, which play a variety of roles in protecting liver, antioxidation, hypoglycemia, antivirus, etc. It has been confirmed that the content of malus hupehensis phloridin in Shennongjia forest region and Yichang is superior. The content of alanine aminotransferase and aspartate aminotransferase in serum is not only the main index for the diagnosis of liver parenchyma damage, but also the typical index of alcoholic liver. Feng Tianyan et al. [5] studied the effect of malus hupehensis on liver fibrosis and verified that total flavonoids of malus hupehensis leaves can reduce the contents of alanine aminotransferase and aspartate aminotransferase in serum, indicating that it has a good protective effect on liver.

Pueraria lobata and tangerine peel are high-frequency drugs used in antialcoholic prescriptions in ancient China Modern pharmacological studies have shown that the occurrence time of drunkenness can be significantly prolonged by intragastric administration of orange peel extract in mice. Moreovr, the two main herbs in the ancient prescription "tangerine peel soup"-tangerine peel and Pueraria lobata-are used to relieve gastrointestinal discomfort after drinking. Jujube and corn cores not only protect the liver and stomach, but also their rich natural sugars help alcohol dehydrogenase decompose alcohol more efficiently and faster.

When this chewable tablet is chewed, it releases total flavonoids, puerarin, flavonoids and limonins at the same time to protect gastric mucosa, enhance acetaldehyde dehydrogenase activity, reduce acetaldehyde damage to the liver, and promote the excretion of alcohol metabolites, thus achieving the effects of anti-alcoholism and liver protection, spleen and stomach. It is worth noting that this product takes the chewable tablet dosage form that young people are used to as the carrier, shortens the distance between traditional Chinese medicine and public life, innovates the chewable tablet market, breaks the current situation of single efficacy and many substitutes in the chewable tablet market, and injects new vitality and business opportunities into the chewable tablet market.

\section{Production process}

\subsection{Experimental materials}

15-30 parts of malus hupehensis, 9-15 parts of Pueraria lobata, 9-15 parts of tangerine peel, 6-12 parts of jujube, 6-12 parts of corn sweet cob, 6 parts of menthol and 20 parts of gum base, wherein, the glue base is made of Pueraria powder and gelatin tablets.

\subsection{Major equipment}

Mold dryers, casters, oscillators, cooling lathes, demoulders, conveying piping systems. 


\subsection{Production process}

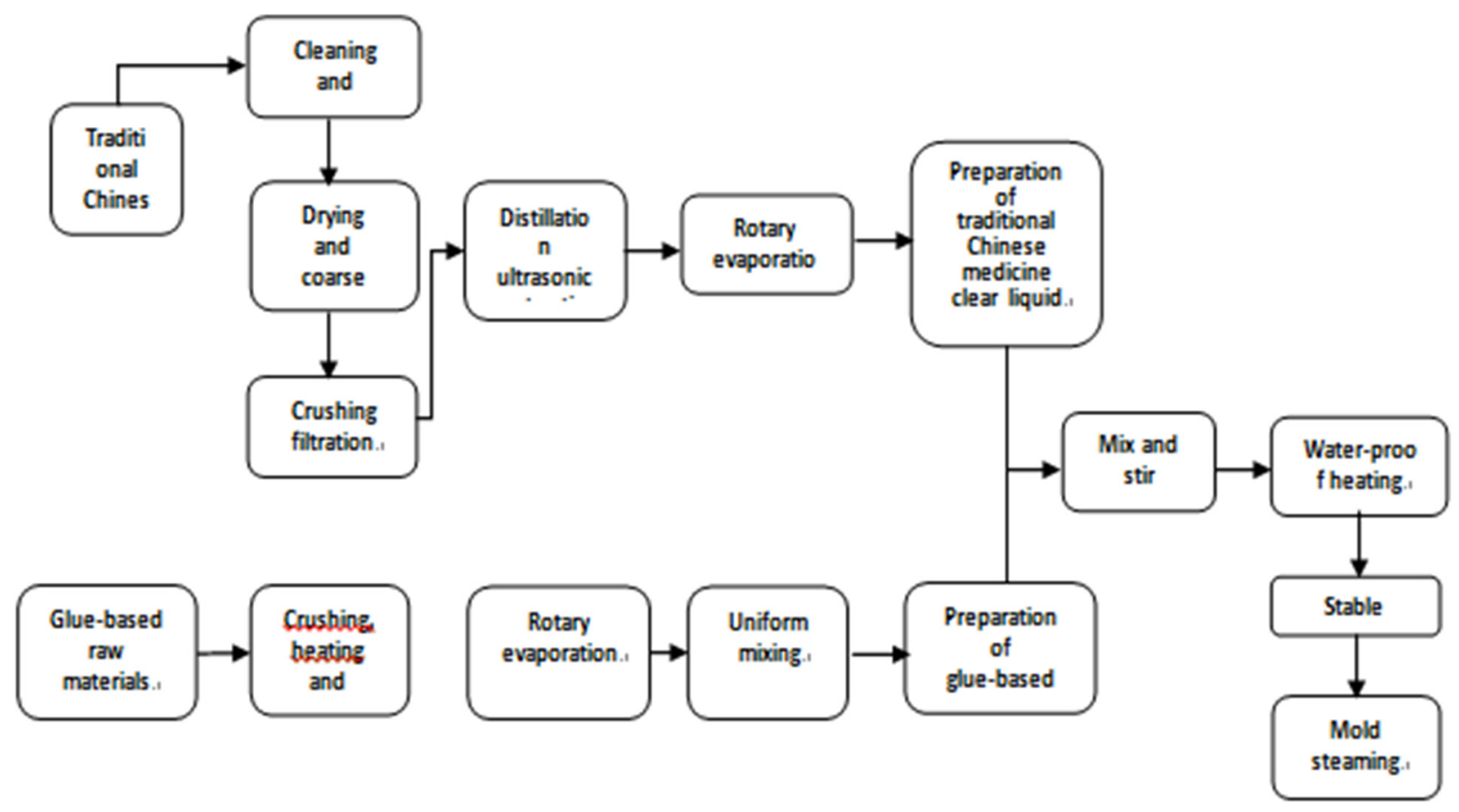

Figure.1 Production process

Step 1. Prepare the clear liquid of traditional Chinese medicine: Wash and soak the Chinese medicinal materials, dry coarsely, crush and filter, distill the original liquid and extract by ultrasonic. Step 2. Prepare the gum base liquid: mix the gum base and menthol, add the clear liquid of traditional Chinese medicine in step 1, and adjust it to an emulsion form to prepare a gum base liquid. Step 3. Prepare gum base drops: heat the gum base liquid of step 2 in a water-resistant manner, stir thoroughly, and mix evenly. Among them, the temperature of waterproof heating is controlled at $50{ }^{\circ} \mathrm{C}$ $\sim 55{ }^{\circ} \mathrm{C}$, which is beneficial to the stable form of glue-based solution. Then into the mold: using a clean eyedropper to drop the glue-based drop into the mold; then steaming and drying: after steaming the mold dripping into the base liquid of the glue, take out the cool demoulding, and then dry the surface water to get the chewable tablets; finally, sterilization and packaging: the chewable tablets are irradiated under the ultraviolet lamp of the ultra-clean worktable for 1 hour, flipped once per $30 \mathrm{~min}$, and then aseptically packaged.

\subsection{Product technical index determination}

For the sensory evaluation method of chewable tablet, the $\mathrm{R} \& \mathrm{D}$ team invited 60 volunteers $(30$ males and 30 females) to evaluate the color, tissue morphology, taste, sweetness persistence, aroma retention and chewing time of the 5 samples. The formula proportion of each sample was different, and Winkids chewing candy in the market was taken as the control group. Its main consideration indexes include: color, which is mainly carried out in terms of whether it is uniform and whether there is the original color of the product; tissue morphology, which is mainly considered in terms of block shape, smooth surface, uniform thickness, stickiness and extensibility after chewing; taste, which is mainly considered from the aspects of stickiness, extensibility and palatability after mastication; sweetness persistence, which is mainly considered in terms of sweetness duration after entrance; and aroma retention, which is mainly considered in terms of the duration of fragrance and whether there is a peculiar smell. The mastication time determines the overall quality of chewable tablet.

This scoring uses a hundred-point scale. The scores of each item are different because of their different degree of impact on product quality. Specifically, color and luster accounted for 15 points, tissue shape accounted for 20 points, taste accounted for 25 points, sweetness persistence accounted for 10 points, fragrance retention accounted for 5 points, and chewing time accounted for 25 points. Finally, count the scores of each formula chewable tablet.

Table.1 Sensory Evaluation Table of Different Samples

\begin{tabular}{|c|c|c|c|c|c|c|c|}
\hline & Color and Luster & Tissue Shape & Taste & Sweetness Persistence & Fragrance Retention & Chewing Duration & Total \\
\hline Sample 1 & 14.12 & 16.35 & 20.23 & 8.64 & 4.43 & 18.26 & 82.03 \\
\hline Sample 2 & 13.56 & 17.02 & 21.55 & 8.35 & 4.03 & 18.55 & 83.06 \\
\hline Sample 3 & 13.02 & 16.98 & 20.90 & 7.56 & 4.58 & 18.62 & 81.66 \\
\hline
\end{tabular}




\begin{tabular}{|c|c|c|c|c|c|c|c|}
\hline Sample 4 & 13.44 & 18.01 & 19.76 & 8.22 & 3.96 & 19.68 & 83.07 \\
\hline Sample 5 & 14.09 & 17.56 & 22.08 & 7.39 & 4.93 & 20.61 & 86.66 \\
\hline Control group & 13.26 & 18.23 & 23.22 & 8.84 & 4.66 & 22.45 & 90.66 \\
\hline
\end{tabular}

\section{Efficacy analysis and evaluation}

A randomized, parallel controlled efficacy study. In the experiment, 70 volunteers (aged 18-60 years old, 80\% male, who had no drinking behavior in the week before the test) were selected to participate in the test. After drinking alcohol at the prescribed dose, all the volunteers developed symptoms such as nausea, vomiting and dizziness. In order to ensure the accuracy of the experimental results, volunteers are not allowed to eat other substances during the anti-alcoholism process, and only drink an appropriate amount of pure water. The specific experimental process is as follows: 70 volunteer subjects were tested to drink ordinary liquor (53 degrees) in the $100-250 \mathrm{ml}$ market. After drinking alcohol, they took 6-8 tablets of this chewable tablet. The degree of improvement of their personal status was evaluated every 45 minutes, and recorded according to 0-3 points, in which $0=$ little improvement, $1=$ slight improvement, 2 = significant improvement, $3=$ complete improvement or basically return to normal state. Various types of data are recorded, as shown in the table below.

Table.2 self-evaluation of the overall efficacy of chewing gum by subjects after using the product

\begin{tabular}{|c|c|c|c|c|c|}
\hline Duration & $45 \mathrm{~min}$ & $90 \mathrm{~min}$ & $135 \mathrm{~min}$ & $180 \mathrm{~min}$ & $225 \mathrm{~min}$ \\
\hline Complete improved (3 points) & 10 & 20 & 32 & 54 & 69 \\
\hline Significant improved (2 points) & 32 & 34 & 22 & 14 & 1 \\
\hline Slightly improved (1 point) & 27 & 16 & 6 & 2 & 0 \\
\hline Little improved (0 points) & 1 & 0 & 0 & 0 & 0 \\
\hline
\end{tabular}

As shown in the table above, in terms of physical discomfort after drinking (including nausea, vomiting, dizziness and headache), 10 people's problems were completely improved, 32 were significantly improved, 27 were slightly improved, and 1 was not significantly improved after 45 minutes of using this anti-alcohol liver protection chewable tablet, while the 69 subjects' drinking state completely improved after using the product and returned to their normal state after 225 minutes of use.

After experimental testing and adjustment of the product ingredient ratio, the best ratio effect has been determined. The test results are as follows.

Table.3 Optimal ingredient ratio test of chewable tablet

\begin{tabular}{|c|c|c|c|c|c|c|c|c|c|}
\hline Research object & \multicolumn{9}{|c|}{ Product ingredient ratio } \\
\hline malus hupehensis & 0 & 1 & 2 & 3 & 3 & 3 & 3 & 3 & 3 \\
\hline Pueraria lobata & 0 & 1 & 1 & 1 & 2 & 2 & 2 & 2 & 2 \\
\hline Tangerine peel & 0 & 1 & 1 & 2 & 1 & 2 & 2 & 2 & 2 \\
\hline Jujube & 0 & 1 & 1 & 1 & 1 & 1 & 2 & 2 & 2 \\
\hline Corn cob & 0 & 1 & 1 & 1 & 1 & 1 & 1 & 2 & 2 \\
\hline Menthol & 0 & 1 & 1 & 1 & 1 & 1 & 1 & 1 & 2 \\
\hline $\begin{array}{l}\text { Average time for subjects to } \\
\text { return to normal }\end{array}$ & $255 \mathrm{~min}$ & $240 \mathrm{~min}$ & $232 \mathrm{~min}$ & $205 \mathrm{~min}$ & $195 \mathrm{~min}$ & $165 \mathrm{~min}$ & $173 \mathrm{~min}$ & $171 \mathrm{~min}$ & $174 \mathrm{~min}$ \\
\hline
\end{tabular}

As can be seen from the above table, with the increase of the proportion of ingredients, the average time for subjects to return to normal state will be shorter, but when more than a certain proportion, the taste of the product will be too sweet, and its acceptance will be affected. The best ratio is malus hupehensis: kudzu root: tangerine peel: jujube: menthol: corn sweet core $=32$ rig 2 purl 1purl. At this time, the taste of the product and the effect of soothing discomfort after anti-alcoholism is relatively the best level.

\section{Conclusion}

For begonia leaves, the folk have a long history of drinking. Modern clinical trials have proved that malus hupehensis leaves have no toxic side effects and can be eaten for a long time. In addition to the hepatoprotective effect, the flavonoid extract of malus hupehensis leaves is also worthy of effective development and application in reducing blood sugar, antioxidation and blood lipids. 
Therefore, it is of extensive research value and market development space to systematically study malus hupehensis leaves, formulate quality standards and develop health food with clear functional components and mechanism. Furthermore, this is of far-reaching significance for the promotion of traditional Chinese medicine culture and the innovative development based on the scientific rigor of traditional Chinese medicine disciplines. What is even more surprising is that through the development of a series of traditional Chinese medicine snack products and innovative food formulations, it is convenient for people to use, and it has drawn the distance between Chinese medicine and the public.

\section{References}

1. Zhou Meili, Li Houtao, Song Junmei. Study on The Preparation Technology of Jingangteng Chewable Tablets[J]. Qilu Pharmaceutical Affairs, 2006(06): 363-365.

2. Zhang Jinping, Wang Heyao, Liu Hua. Research on The New Technology of Direct Compression of Aspirin Tablet Powder[J]. Chinese Journal Of New Drugs,2003(01):45-46.

3. Zhang Huixiang, Liu Linwei, Yang Shijun. Research Progress In Anti-Alcoholism Medicines And Health Foods[J]. Natural Products Research And Development, 2002(02):83-86.

4. Sun Yong, Peng Mingli. Research Progress of Curcumin and Its Derivatives in The Prevention and Treatment of Liver-Related Diseases[J]. Acta Pharmaceutica Sinica, 2014,49(11):1483-1490.

5. Feng Tianyan, Wang Yunzhi, Zhou Jigang, Deng Gigai, Zhang Qiaoyin, Zou Kun. Malus Hupehensis Leaf Total Flavonoids Anti-Ccl_4 Induced Liver Fibrosis In Rats[J].Pharmacology And Clinics Of Chinese Materia Medica,2012,28(02):72-76. 